Journal of Engineering and Applied Sciences 14 (Special Issue 8): 10216-10225, 2019

ISSN: 1816-949X

(C) Medwell Journals, 2019

\title{
Accurate Localization System using UWB Technology
}

\author{
Ekhlas K. Hamza Hanan, Hussain, Al-Asady and Areej Alaa Hassan \\ Department of Control and System Engineering, University of Technology Baghdad, \\ Baghdad, Iraq
}

\begin{abstract}
Localization is one of the motivating research areas in wireless networks, especially when a system is required to operate in a harsh environment. Many researchers have agreed that utilizing larger numbers of anchor nodes should increase positioning accuracy. The majority of localization system algorithms use two approaches; ranging and positioning. For ranging (RSS) uses power of the arriving signals to identify the gap between transmitters and a receiver and Time of Arrival (TOA) uses the time required by a signal to travel between a transmitter and a receiver to identify distance, thus, it requires synchronization. In this study, TOA ranging technique is used for different estimation nods. Simulation results are performed using MATLAB, while the actual results are obtained using EVK1000 device for indoor positioning platform. The estimation algorithm implemented uses the Linearized Least Square (LLS) method. It was studied in details and a testing area in an indoor environment was prepared to suit LOS, NLOS and combined situations. The collected data showed that sometimes the accuracy of two anchor nodes system was better than the one using three nodes. This may allow utilizing smaller number of anchors while maintaining the required accuracy at a lower cost.
\end{abstract}

Key words: Wireless systems, wireless sensors network, indoor positioning system, ultra-wide band signal, range-based, maintaining

\section{INTRODUCTION}

Localizing objects has been of considerable importance for navigation and general mankind activities for quite some time (Perttula et al., 2014). At present, Global Positioning System (GPS) can provide object localization services with accuracy of few meters only (Perttula et al., 2014; Yayan et al., 2015). However, with regards to localizing or navigating objects in an indoor environment, there is a need for greater accuracy (Yayan et al., 2015). While systems comes to be exceedingly standard in latest years, there is yet amore to be done to improve them. Currently, there are several indoor positioning systems that have been successfully implemented in different tracking and navigation applications (Zekavat and Buehrer, 2011). The systems rely on RF inter-nodes where distance estimation is evaluated by calculating transit time of signals traveling between pairs of nodes (Zekavat and Buehrer, 2011). Given the distance estimates between few nodes designated as Anchor Nodes (ANs) and a Target Node (TN) the TN's position can be estimated (Yayan et al., 2015).

There are wide varieties of localization systems and techniques, a localization system with an accuracy better than $1 \mathrm{~m}$ is required for human movement localization (Pahlavan et al., 2002). These systems are the key enabler for providing position specific information on mobile devices in a building. It measures the distance between a point of unknown position (Mobile $M$ ) and three points at known positions as shown in Fig. 1 (Yayan et al., 2015; Pahlavan et al., 2002).

This study includes a summary of common radio technologies that can be implemented in an (ips). Several systems, techniques and procedures which investigated or assessed of the research. Additionally, information on how to configure and test the (EVK1000) evaluation board from DecaWave Inc. The prime objective of this work was to design an indoor positioning system for sport tracking through selecting proper methods, developing the necessary algorithms and experimentally evaluating the end system. The EVK1000 board was the main piece of technology necessary to develop the indoor localizing system.

Schemes for indoor positioning systems: There are four technique are descript in following subsuction. The main ranging technique used for indoor positioning in this thesis is time of arrival TOA and other popular indoor positioning techniques are outlined and briefly explained in the following subsections (Pahlavan et al., 2002; Gezici, 2008). The DecaWave or TREK1000 system uses a two way ranging TOA technique which involves precise timing, so, DacaWave1000 uses SDS-TOA technique which allows for a complete ranging and minimizes ranging error compared to other positioning techniques (Fuchs et al., 2011; Ali and Omar 2005).

Corresponding Author: Ekhlas K. Hamza, Department of Control and System Engineering, University of Technology Baghdad, Baghdad, Iraq 


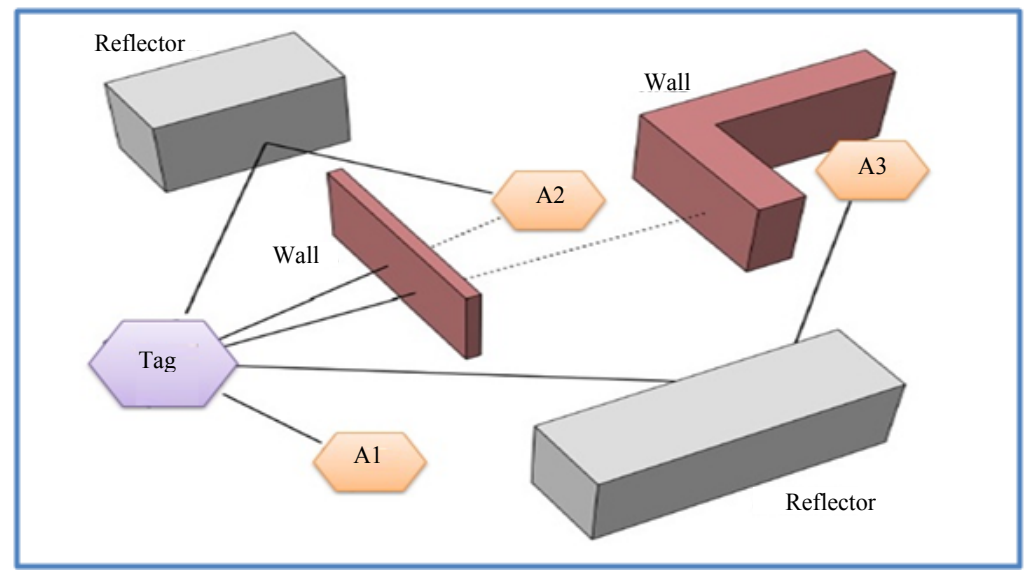

Fig. 1: Multipath problems of traditional ultra-wideband radio signal based positioning (Pahlavan et al., 2002)

Time of Arrival (TOA): A technique that comprises determining the distance between two nodes by using the time was TOA, it yields for the radio signal to move between the transferring and receiving node (Yan, 2010). A synchronized time base to accurately timestamp packets requires for both the anchor and the tag in TOA. If the anchor and target are not time synchronized, two-way ranging is useful such as in the DecaWave, TREK1000 system where the ranging communication is sent between the anchor and the tag, then back to the anchor to precisely compute the signal time of flight (Fuchs et al., 2011; Yan, 2010). The separated distance from an anchor to focus node is acquired by multiplying the estimated TOA with the speed of light (Yan, 2010). This estimated distance is very susceptible to timing error, even a very small percentage of error in timing can result in a large error in the value of calculated distance (Liu et al., 2007).

Ultra-Wideband technology (UWB): Another radio frequency method implemented in indoor positioning systems is the ultra-wide band technique which is slightly different from the previously discussed scheme (Perttula et al., 2014). The frequency range $(3.14-10.6 \mathrm{GHz})$ of an UWB radio implemented in a localization system. It is characterized by a data rate greater than $500 \mathrm{MHz}$ making it capable of establishing multipath communication. The UWB radio system is featured by low transmitting power with pulse duration varying from few picoseconds to nanoseconds (Yayan et al., 2015; Albaidhani et al., 2016). This low power of transmission supports the transmitted not is disturbed by the noisness of reflected waves from obstacles on a line of sight. It is possible to place reference points outside the building this makes UWB technology capable of providing more reliable performance for object localization applications than other radio signal technologies implemented for indoor localization systems (Albaidhani et al., 2016).
The advantages gained from implementing UWB: The UWB technology has been employed in indoor positioning systems by several leading companies such as time domain and DecaWave. UWB provides several advantages over other rival IEEE wireless protocols. UWB communication systems are featured by the following advantages (Yan, 2010; Anonymons, 2004):

- It can operate alongside other systems that operate using IEEE wireless spectrums

- It is characterized by a very large channel capacity

- It can provide acceptable performance in noisy environments and operates with low SNR

- It implements low transmission power which makes detecting and intercepting very difficult

- It is characterized with high resistance to jamming

- It delivers high performance in multipath channels

Work parameters: The working parameters are divided into two parts: (Anonymons, 2015) hardware and software.

Hardware requirements: The DecaWave (DW1000) is implemented in the designed object localizing system, the kit contains the following parts:

- $2 \times \mathrm{EVB} 1000$ boards

- $2 \times$ antennae

- $2 \times \mathrm{USB} 2.0$ cable

- $2 \times$ power leads

- $2 \times$ perspex stands

The EVB1000 evaluation board is of $7 * 7 \mathrm{~cm}$ size. Figure 2 shows its two sides with its main components identified. There is an LCD display on its front side it is used to show ranging information and the operating mode of the board. The DIP Switch (S1) allows the user to 
(a)

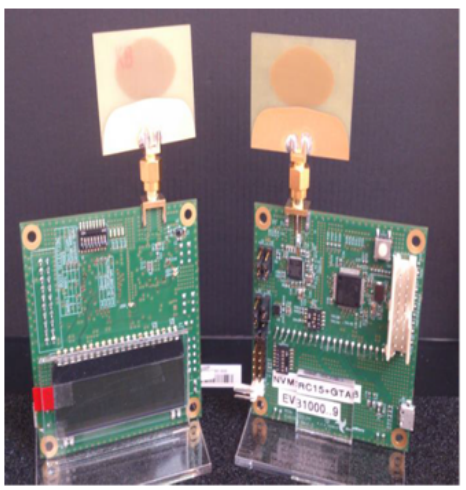

(b)

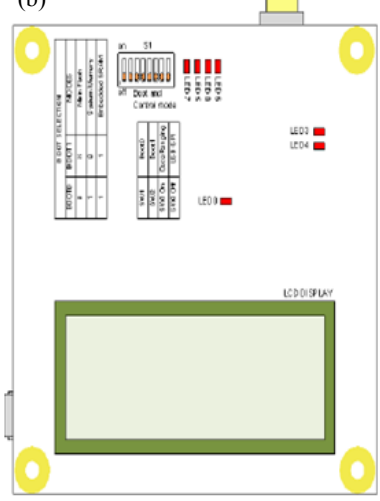

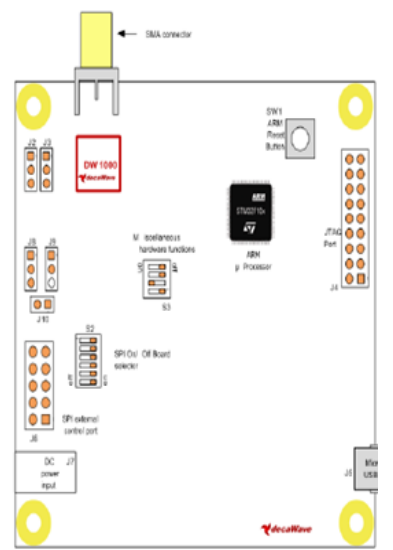

Fig. 2(a, b): Main components of the EVB1000 (Anonymons, 2015)

select the operating mode of the EVB1000 board. There are also a number of LED indicators that provides the user with useful information.

Software requirements: The CooCox CoIDE is a software solution that enables users to create and compile $\mathrm{C}$ code for ARM architecture based micro controllers and devices. It supports a large number of boards and chips for which the installation and configuration program can be written and modified using this development platform. The CoIDE Software modules were downloaded from the Coocox website (coocox.org).

The real and estimated location for anchor and tag nodes: The following tables show the location of fix anchor nodes (A0-A2) with the numbers of tag nodes for LOS and NLOS measurement as following: $(\mathrm{x}, \mathrm{y})$ represented the real location value of indoor environment, $\operatorname{Avg}(\mathrm{x}), \operatorname{Avg}(\mathrm{y})$ represented the stimate $(\mathrm{x}, \mathrm{y})$ for average of hundreds management values read by EVK1000 kite. Error $(x, y)$ represented the error value for $(x, y)$ find by subtract real value of $(x, y)$ from the estimated value of $(x$, y) which is Useful when drawing the flowchart of localization system.

The position error find by using the following equation for different nodes and for both LOS and NLOS.

$$
\text { position error }=\sqrt{\text { error }(x)^{2}+\operatorname{error}(y)^{2}}
$$

\section{MATERIALS AND METHODS}

Testing: A testing procedure is laid down in order to determine the obtainable measurement accuracy when operating the designed object localization system while using two and three anchor nodes in different testing setup conditions. There are several factors that are considered to have adverse effects on measurement accuracy which must be taken into consideration while evaluating system performance as they have a strong influence on operational accuracy figures. In this study, only the issue of distance estimation was covered. It was tested for two and three anchor nodes with one tag (mobile node). To measure the positioning accuracy, two and three EWB1000 evaluation boards were used in different indoor locations with LOS and NLOS cases. The localization algorithm used in this work is Linear Least Square (LLS) method. It goes on as follows: Let $\mathrm{x}_{1}$ and $\mathrm{y}_{1}$ represent the coordinates of the anchor node 1 (reference node), xi and yi $(i=2 \mathrm{~m})$ are the other anchor nodes coordinates and $\mathrm{x}, \mathrm{y}$ are the mobile station coordinates. Initially let $\mathrm{x}_{1}=\mathrm{y}_{1}=0$. Then the Euclidian distance between the mobile station and every anchor node as below:

$$
\begin{aligned}
& \mathrm{r}_{1}^{2}=\left(\mathrm{x}-\mathrm{x}_{1}\right)^{2}+\left(\mathrm{y}-\mathrm{y}_{1}\right)^{2} \\
& \mathrm{r}_{\mathrm{i}}^{2}=\left(\mathrm{x}-\mathrm{x}_{2}\right)^{2}+\left(\mathrm{y}-\mathrm{y}_{2}\right)^{2} \\
& \mathrm{r}_{\mathrm{i}+1}^{2}=\left(\mathrm{x}-\mathrm{x}_{3}\right)^{2}+\left(\mathrm{y}-\mathrm{y}_{3}\right)^{2} \\
& \mathrm{r}_{\mathrm{m}}^{2}=\left(\mathrm{x}-\mathrm{x}_{\mathrm{m}}\right)^{2}+\left(\mathrm{y}-\mathrm{y}_{\mathrm{m}}\right)^{2}
\end{aligned}
$$

The problem aboveis that Least Square (LS) is a nonlinear problem. To linearize it the following steps are performed; subtract every distance equation $\left(\mathrm{r}_{\mathrm{i}}\right)$ with the reference distance $\left(r_{1}\right)$.

$$
\begin{gathered}
\mathrm{A}=\left[\begin{array}{cc}
2 \mathrm{x}_{2} & 2 \mathrm{y}_{2} \\
2 \mathrm{x}_{3} & 2 \mathrm{y}_{3} \\
\vdots & \\
2 \mathrm{x}_{\mathrm{m}} & 2 \mathrm{y}_{\mathrm{m}}
\end{array}\right] \\
\mathrm{b}=\left[\begin{array}{c}
\mathrm{b}_{2} \\
\mathrm{~b}_{3} \\
\vdots \\
\mathrm{b}_{\mathrm{m}}
\end{array}\right]
\end{gathered}
$$




$$
\begin{gathered}
\mathrm{b}_{2}=\mathrm{x}_{2}^{2}+\mathrm{y}_{2}^{2}+\mathrm{r}_{1}^{2}-\mathrm{r}_{2}^{2} \\
\mathrm{~b}_{2}=\mathrm{x}_{3}^{2}+\mathrm{y}_{3}^{2}+\mathrm{r}_{1}^{2}-\mathrm{r}_{3}^{2} \\
\mathrm{~b}_{\mathrm{m}}=\mathrm{x}_{\mathrm{m}}^{2}+\mathrm{y}_{\mathrm{m}}^{2}+\mathrm{r}_{1}^{2}-\mathrm{r}_{\mathrm{m}}^{2} \\
\mathrm{X}=\left[\begin{array}{l}
\mathrm{x} \\
\mathrm{y}
\end{array}\right]=\left(\mathrm{A}^{\mathrm{T}} \mathrm{A}\right)^{-1} \mathrm{~A}^{\mathrm{T}} \mathrm{b}
\end{gathered}
$$

\section{RESULTS AND DISCUSSION}

Conducting of the firstly experiment: In this experiment the DecaWave's device is used to calculate the $(x, y)$ and find the location of any node in the indoor localization system. Because DW1000® is designed to determine only ranging operation (detection range and communication range). It is not designed to find the location of nodes $(x, y)$. The following steps describe the experiment procedure in more details:

Step 1; reprograming the DW1000®kit: The DecaWave $1000 \AA$ is designed to compute only ranging data (FSL, RSL), not aimed to find the position of nodes $(\mathrm{x}, \mathrm{y})$, using CooCox program the position of nodes is computed using special equation and code.

Step 2; preparing the work environment: $A(6 \times 7 \times 3 \mathrm{~m})$ office is chosen as a working environment. The purpose is to display the location of MS (human walk) in four positions and two situations: LOS or NLOS (with different heights) as shown in Fig. 3.
Step 3; structure of nodes and measurements: Two fixed positions (A1, A2) were chosen as fixed points as anchor nodes. One moving point is chosen as a tag node which is denoted as $\mathrm{Ti}, \mathrm{i}=0,1, \ldots, 7$. The location coordinate of anchor nodes are sent via. ethernet to the tag node. The tag node is connected to $\mathrm{PC}$ to send continues position coordinates, First Signal Level (FSL), (RSL) and any probable information. The initial position of the tag is chosen to achieve two conditions (LOS and NLOS) as represented in the following Table 1-4.

Step 4; simulating results using MATLAB: An m-file is written to build the LMSE localization algorithm. Two types of input date are processed: actual position of the tag (taken directly by the user) and the estimation position as sent by the tag node. The length of the received data is huge, thus, the program takes its average. Execute the code results in two outputs: the average curves of estimated and real positions and error position. They are plotted to analyze and compare the results. The comparison is between a movement of the MS and the position of this mobile with two anchor nodes for real and estimates values for LOS and NLOS with different height as shown in Fig. 4 and 5.

Conducting of the secondly experiment (real environment): The goal of the secondly experiment is to show in what way the number of nodes of DW1000® decided the high accuracy of MS location in indoor environment. It will be shown that the increase of the

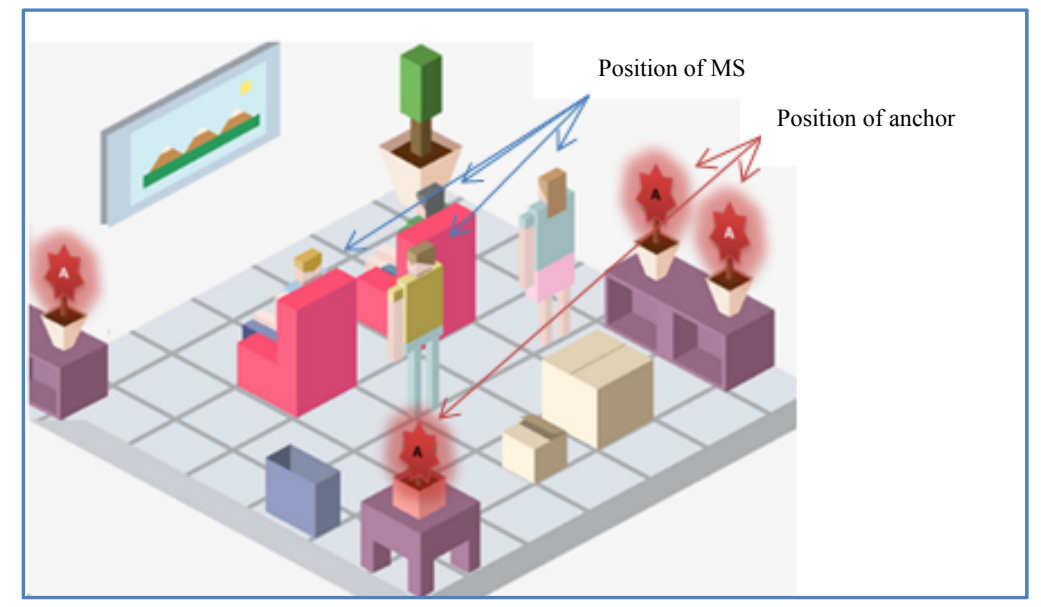

Fig. 3: Location representations for LOS condition using two or three anchor nodes

Table 1: Results of two anchor nodes and five tag nodes (LOS measurement)

\begin{tabular}{llllllllll}
\multicolumn{2}{l}{ Anchor } & X & Y & Tags & X & Avg(x) & Y & Avg (y) & Error \\
\hline A0 & 0 & 0 & T0 & 5.1 & 5.098993 & 8.4 & 8.548901 & 0.148904 \\
& - & - & T1 & 5.8 & 5.543362 & 5.4 & 6.123749 & 0.662693 \\
& - & - & T2 & 6.6 & 6.546593 & 6.26 & 6.975261 & 0.770571 \\
A1 & 1 & 1.8 & T3 & 4.5 & 4.429836 & 3.6 & 3.590375 & 0.923386 \\
& - & - & T4 & 1.6 & 1.547866 & 4.2 & 3.987542 & 0.938471 \\
\hline
\end{tabular}


Table 2: Results of three anchor nodes andeight tag nodes (LOS measurement)

\begin{tabular}{lllllllll}
\hline Anchor & X & Y & Tags & X & Avg(x) & Y & Avg (y) & Error \\
\hline A0 & 0 & 0 & T1 & 7.2 & 7.007381 & 9 & 8.929779 & 0.20502 \\
& - & & T2 & 0.6 & 0.563142 & 8.4 & 8.645489 & 0.24824 \\
& - & & T3 & 0.6 & 0.473673 & 7.2 & 7.277158 & 0.148027 \\
A1 & 1 & 1.8 & T4 & 1.2 & 1.204593 & 9.6 & 9.686605 & 0.086726 \\
& - & & T5 & 1.8 & 1.772573 & 6 & 6.511426 & 0.512161 \\
& - & & T6 & 2.1 & 2.291997 & 9.9 & 10.28439 & 0.429669 \\
A2 & 4.8 & 0 & T7 & 3 & 3.001370 & 4.8 & 5.0132 & 0.213205 \\
& - & & T8 & 4.2 & 4.271505 & 4.2 & 4.215813 & 0.073232 \\
\hline
\end{tabular}

Table 3: Results of three anchor nodes and four tag nodes (NLOS measurement)

\begin{tabular}{lllllllll}
\hline Anchor & X & Y & Tags & X & Avg (x) & Y & Avg (y) & Error \\
\hline A0 & 0 & 0 & T0 & 5.1 & 5.098993 & 8.4 & 8.548901 & 0.148904 \\
A1 & 1 & 1.8 & T1 & 5.8 & 5.835362 & 5.4 & 6.061749 & 0.662693 \\
A2 & 4.5 & 0 & T2 & 6.6 & 6.263593 & 6.26 & 6.893261 & 0.770571 \\
- & - & - & T3 & 4.5 & 5.423336 & 3.6 & 3.590375 & 0.923386 \\
\hline
\end{tabular}

Table 4: Results of three anchor nodes and four tag nodes (NLOS with high $(7.8 \mathrm{~m}$ ))

\begin{tabular}{lllllllll}
\hline Anchor & X & Y & Tags & X & Avg (x) & Y & Avg (y) & Error \\
\hline A0 & 0 & 0 & T1 & 1.6 & 1.695619 & 3.5 & 3.355949 & 0.637093 \\
A1 & 4.2 & 0 & T2 & 1.0 & 0.788774 & 2.3 & 2.901058 & 0.172898 \\
A2 & 1.2 & -1.8 & T3 & 2.2 & 2.045348 & 4.7 & 5.163153 & 0.488291 \\
- & - & - & T4 & 3.4 & 3.407954 & 5.9 & 6.074751 & 0.174932 \\
\hline
\end{tabular}
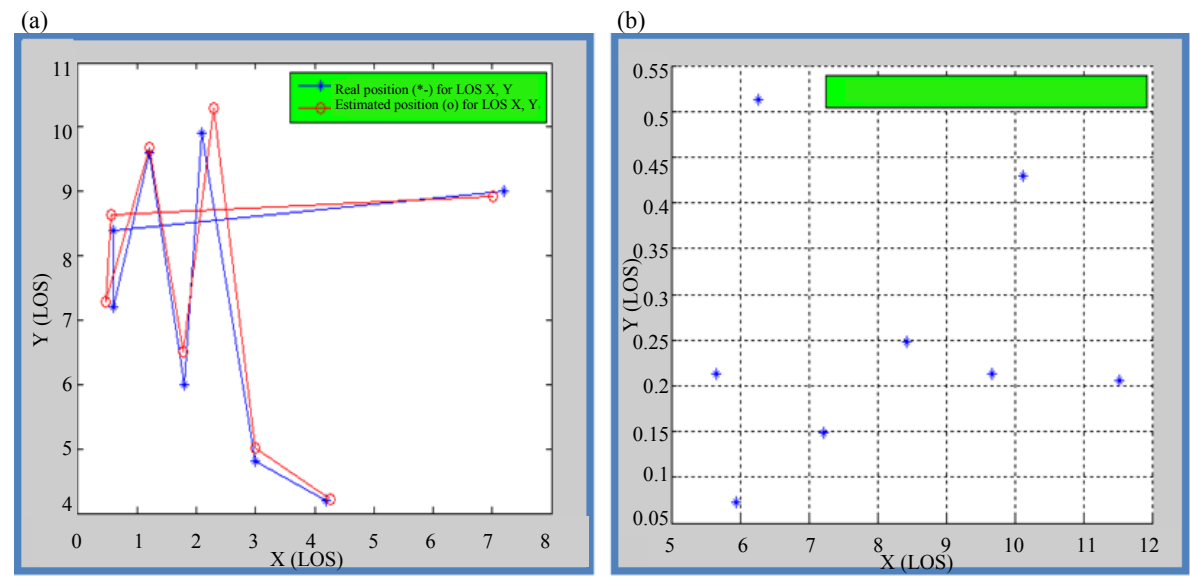

Fig. 4(a, b): The real and estimated position and error position for LOS condition
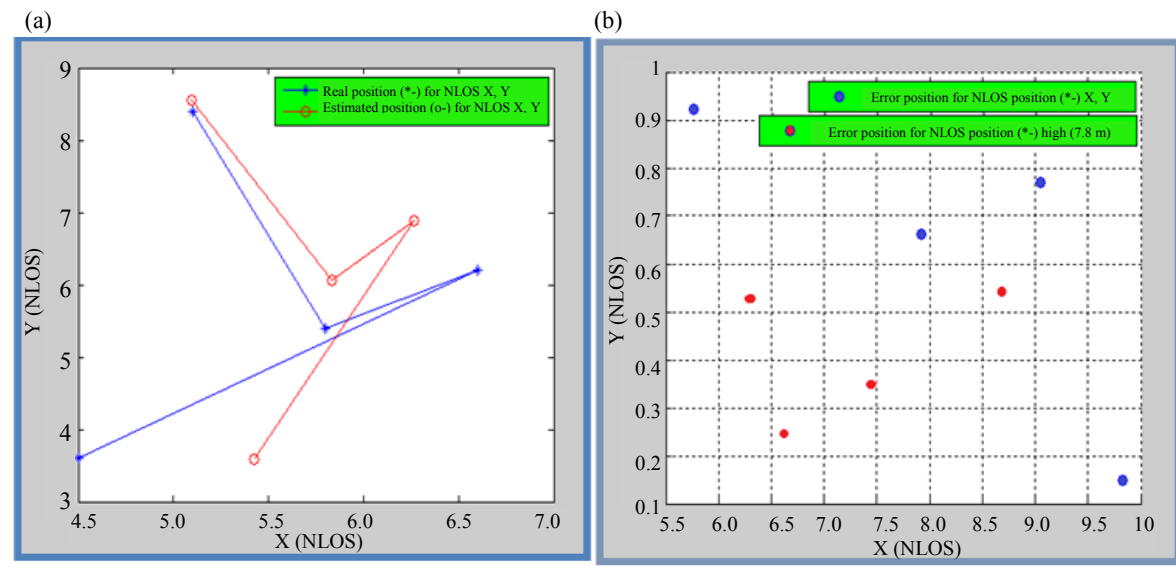

Fig. 5(a, b): The real and estimated position and error position for NLOS condition 
number of nodes could not give the better accuracy and help to find the specific location of MS.The following steps describe the experiment procedure in more details:

Step 1; preparing the work environment: $A(9 \times 6 \times 3 \mathrm{~m})$ office is chosen as a working environment. The purpose is to display the location of MS (human walk) in different positions with two situations: LOS or NLOS, the position of the anchors is fixed while the tag can move freely within the line of sight in all three dimensions. Figure 6 shows the structure of the experiment space, position of the anchors and the region available for positioning, the set of points and a path to obtain range measurements for experiments, respectively. These points and path used for two and three nodes under both of LOS and NLOS condition.

Step 2; the experiment scenarios: The following scenarios displays the real position of MS computed mathematically, estimated position determined by
DW1000® measurements and explain how a location of error and moving the MS decided which scenario give the butter accuracy for different number of anchor nodes.

Figures 7 and 8 illustrate the expected location of MS by two nodes is better than three node for as the error of the indicated position from the estimated ranging for two nodes is less than the error calculated by LMSE method for three nodes .

Step3; structure of nodes and measurements: Two or three fixed positions (A1-A3) were chosen as fixed points as anchor nodes. One moving point is chosen as a tag node which is denoted as $\mathrm{Ti}, \mathrm{i}=0,1, \ldots, 8$. The location coordinate of anchor nodes are sent via. ethernet to the tag node. The initial position of the tag is chosen to achieve both conditions (LOS and NLOS). The next tables give a summary of the data together with the results (error of position) during the movement of MS in different locations in the building (Table 5 and 6 ).

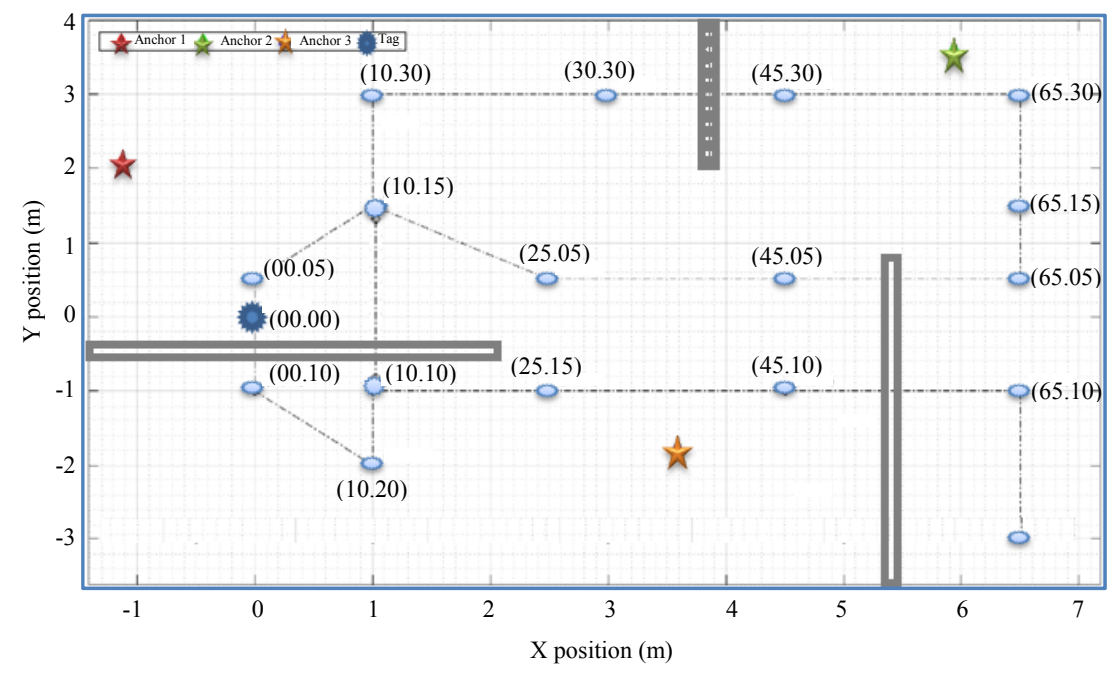

Fig. 6: Measurement points and path used for experiments, wireless based positioning

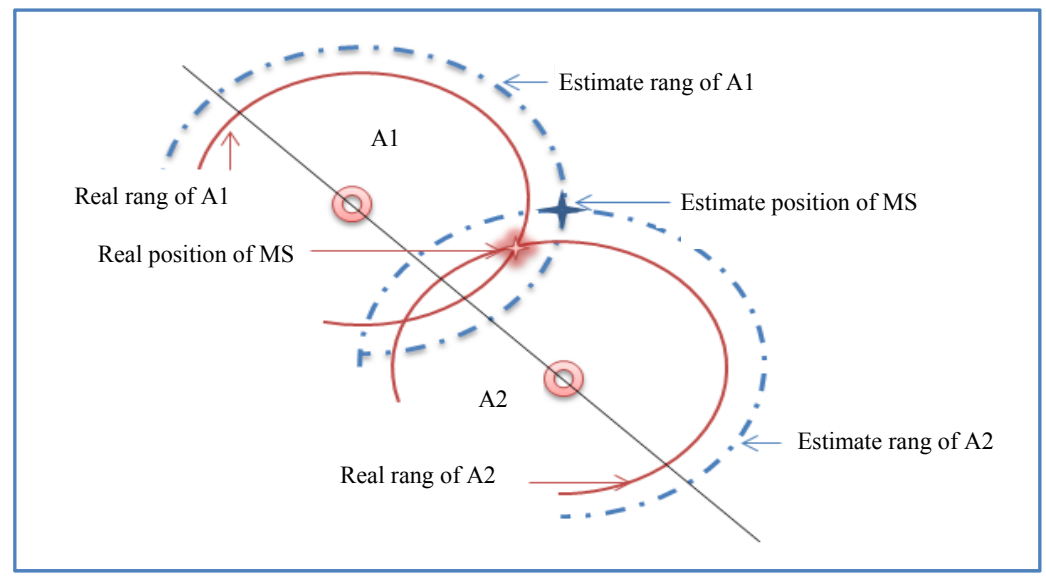

Fig. 7: The real and estimate ranging for two anchor node (A1, A2) 


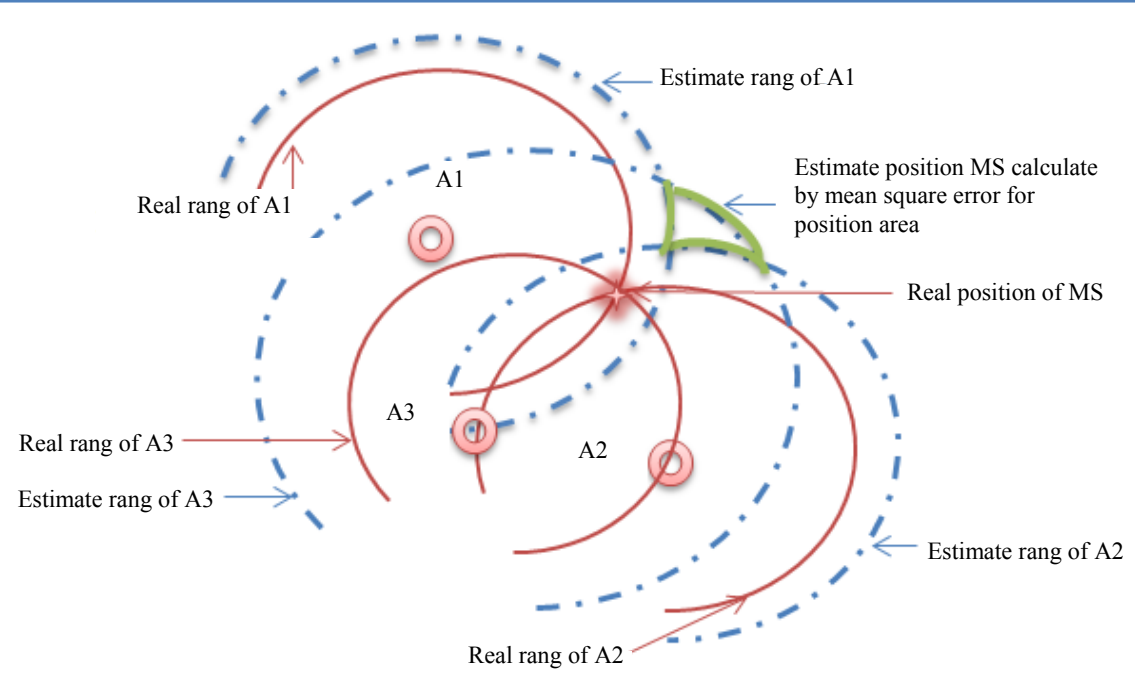

Fig. 8: The real and estimate ranging for three anchor node (A1-A3)
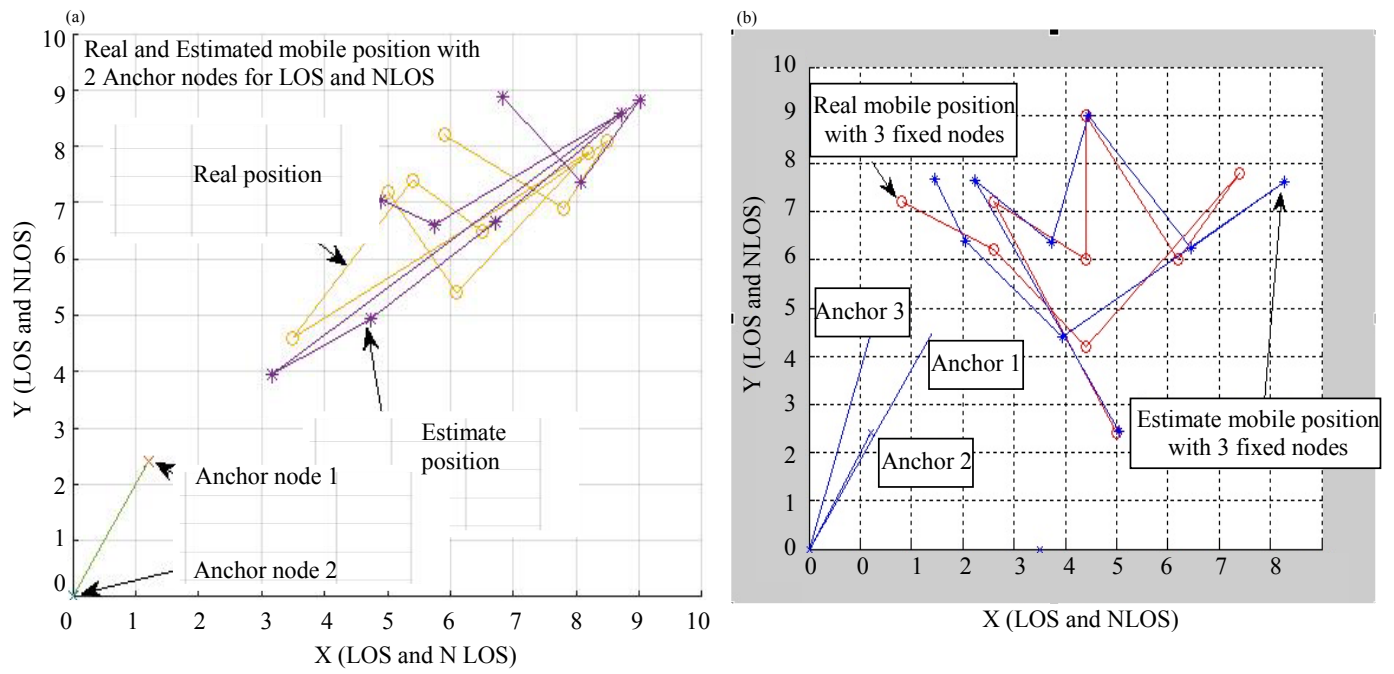

Fig. 9(a, b): Deferent mobile position with 2 and 3 Anchor nodes for LOS and NLOS

Table 5: Results of 3 anchor nodes and 9 tag nodes (LOS measurement)

\begin{tabular}{lllllllllll}
\hline Anchor S & X & Y & Tags & X real & Estimate (x) & Error (x) & Y & Estimate (y) & Error (y) & Error position \\
\hline A0 & 0 & 0 & T0 & 5 & 4.898779 & -0.1012 & 7.2 & 7.052748 & -0.1473 & 0.178714 \\
& - & - & T1 & 6.1 & 5.74545 & -0.3545 & 5.4 & 6.606733 & 1.2067 & 1.257694 \\
& - & - & T3 & 8.2 & 8.716033 & 0.5160 & 7.9 & 8.586433 & 0.6864 & 0.858721 \\
A1 & 1.2 & 2.4 & T4 & 3.5 & 3.160067 & -0.3399 & 4.6 & 3.946767 & -0.6532 & 0.736344 \\
& - & - & T4 & 5.4 & 6.726133 & -0.6576 & 7.4 & 8.667833 & -2.4573 & 2.543769 \\
& - & - & T5 & 5 & 4.742367 & 0.2261 & 5 & 4.942733 & 0.1678 & 0.281564 \\
& - & - & T6 & 8.5 & 9.016133 & 0.5161 & 8.1 & 8.832117 & 0.7321 & 0.895729 \\
& - & - & T7 & 7.8 & 8.080633 & 0.2806 & 6.9 & 7.358667 & 0.4587 & 0.531179 \\
& - & - & T8 & 5.9 & 6.826133 & 0.9261 & 8.2 & 8.877833 & 0.6778 & 1.147638 \\
\hline
\end{tabular}

Step 4; experiment results: The movement of the MS and its position with three anchor nodes of real and estimated values for LOS and NLOS to different tags are shown in Fig. 9. The pictures in Fig. 10 shows the location of the three anchor node and the mobile station with three anchor node and the mobile station with different values of error in two situations that were used to find the mean position error for two and three anchor 


\begin{tabular}{|c|c|c|c|c|c|c|c|c|c|c|}
\hline Anchor s & $\mathrm{X}$ & $\mathrm{Y}$ & Tag & $\mathrm{X}$ real & Estimate(x) & Error (x) & $\mathrm{Y}$ & Estimate (v) & Error (v) & Error position \\
\hline \multirow[t]{3}{*}{$\mathrm{A} 0$} & 0 & 0 & T0 & 6 & 6.047832 & 0.0478 & 2.4 & 2.428928 & 0.0289 & 0.055857 \\
\hline & - & - & $\mathrm{T} 1$ & 3.6 & 3.226254 & -0.3737 & 7.2 & 7.644155 & 0.4442 & 0.580487 \\
\hline & - & - & $\mathrm{T} 2$ & 5.4 & 4.729158 & -0.6708 & 6 & 6.354133 & 0.3541 & 0.758525 \\
\hline \multirow[t]{3}{*}{ A1 } & 1.2 & 2.4 & $\mathrm{~T} 3$ & 5.4 & 5.458767 & 0.0588 & 9 & 8.980433 & -0.0196 & 0.061981 \\
\hline & - & - & $\mathrm{T} 4$ & 7.2 & 7.456433 & 0.2564 & 6 & 6.241677 & 0.2417 & 0.352363 \\
\hline & - & - & $\mathrm{T} 5$ & 8.4 & 9.284433 & 0.8844 & 7.8 & 7.611651 & -0.1883 & 0.904224 \\
\hline \multirow[t]{3}{*}{$\mathrm{A} 2$} & 4.5 & 0 & T6 & 5.4 & 4.933967 & -0.4660 & 4.2 & 4.396396 & 0.1964 & 0.505697 \\
\hline & - & - & $\mathrm{T} 7$ & 3.6 & 3.05445 & -0.5455 & 6.2 & 6.377133 & 0.1771 & 0.573528 \\
\hline & - & - & T8 & 1.8 & 2.454767 & 0.6548 & 7.2 & 7.684565 & 0.4846 & 0.814617 \\
\hline
\end{tabular}
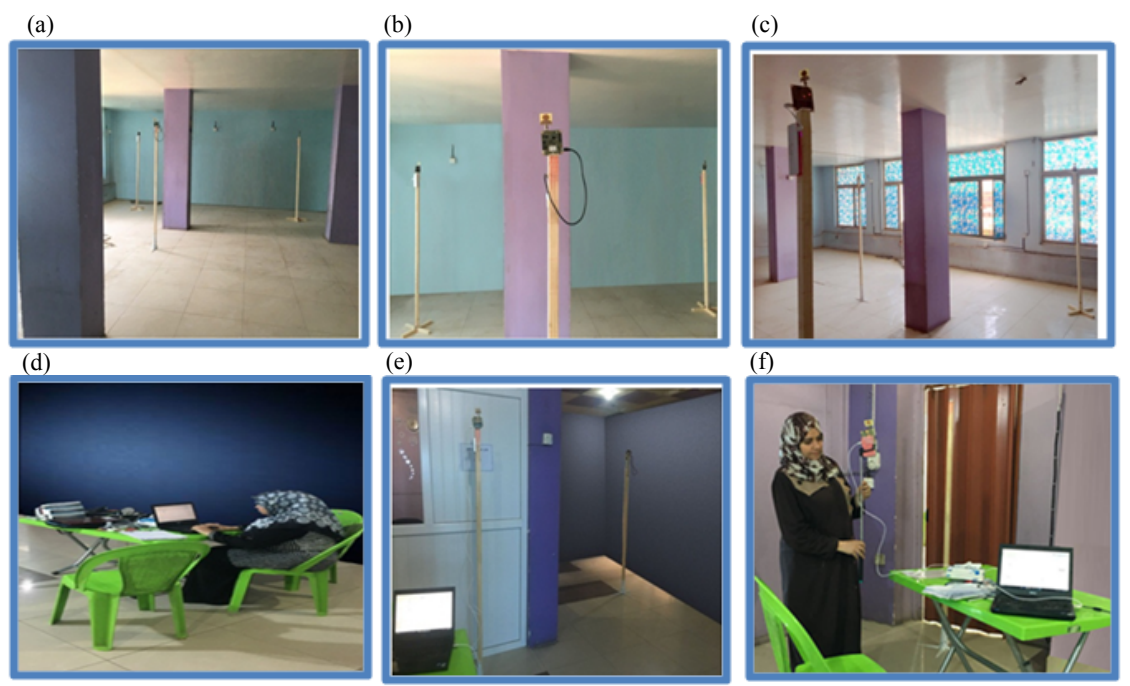

Fig. 10(a-f): The statement location of anchor and tag nodes
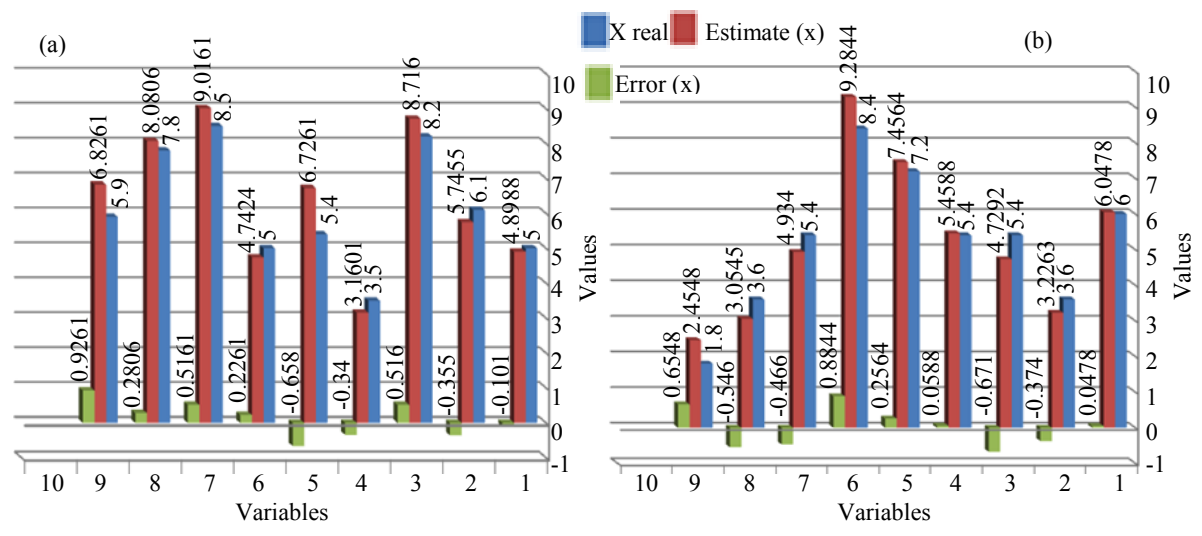

Fig. 11(a, b): The value of $\mathrm{X}$ with two and three anchors node for LOS and NLOS

nodes object localization system. The results obtained from the experimental work suggests that not always three anchor nodes system could be better in estimating target position than the one with two nodes. About 0252074496 the following (Fig. 11-13) represent the location for two and three anchor nodes with two different situation (LOS or NLOS) to show and compere the different between the real and estimate values of $\mathrm{x}$ and $\mathrm{y}$ and the error position for (two and three) anchor nodes. About 0252074496 the results shown in Fig. 14 indicates that in some points using two anchor nodes object localization system can perform better than the one using three anchor nodes in find the location of a mobile station with less error. Form observing these experimental results one can s uggest that using two anchor nodes yields better accuracy than the one using three anchor nodes to find the location of (MS) while at the same time reduces hardware requirements which results in a lower priced system. 

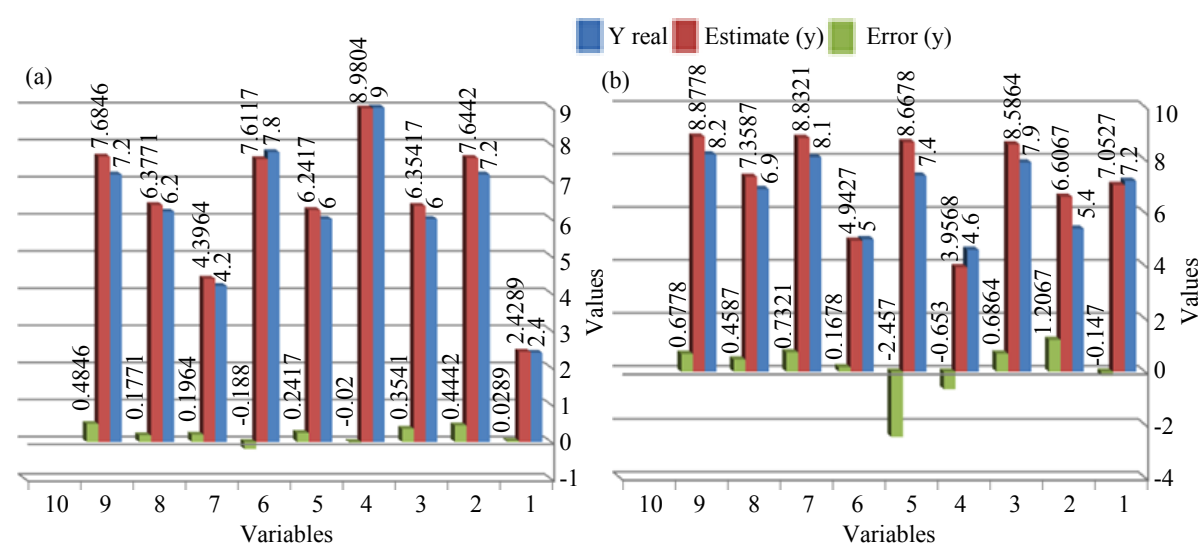

Fig. 12(a, b): The value of $\mathrm{Y}$ with 2 and 3 anchor nodes for LOS and NLOS
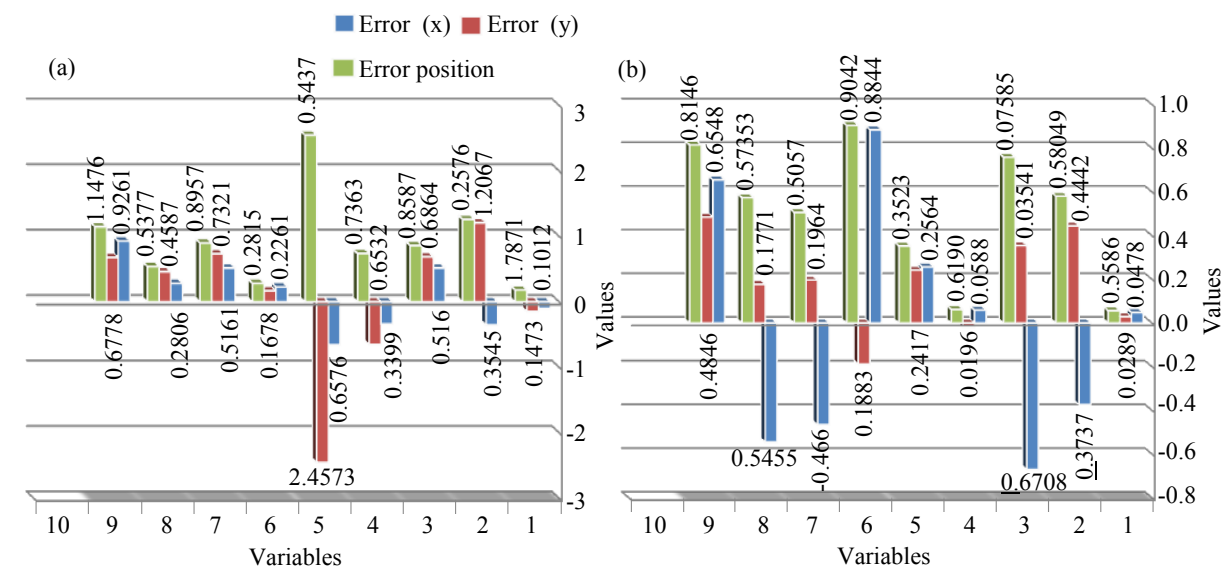

Fig. 13(a,b): The value of Error with 2 and 3anchor nodes for LOS and NLOS

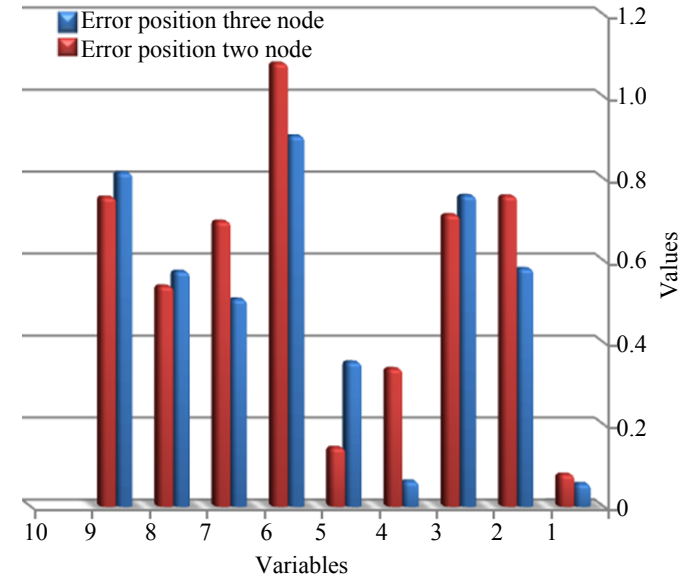

Fig. 14: Comparing error values between two and three anchor nodes for LOS and NLOS

\section{CONCLUSION}

The experimental evaluation of the simulated and implemented UWB localization system were presented in this section. The DecaWave EVK1000 UWB transceiver units were deployed in the implemented UWB localization system. During the conducted experimentation it was found that this device required custom software and a better antenna to suit the requirements of the developed system and to deliver better accuracy.

The purpose of the first set of experiments was to demonstrate that the DecaWave UWB product which was originally designed to deal only with ranging applications could be adapted to find the location of nodes $(\mathrm{x}, \mathrm{y})$ where the DecaWave device (EVK1000 board) was used to calculate the $(x, y)$ and find the location of any node in an indoor localization system by modifying its original program.

The purpose of the second set of experiments was to find out how the number of EVK1000 boards decides the accuracy figure of an indoor localization of a mobile station. The obtained experimentation results indicated that increasing the number of EVK1000 boards didn't help in increasing the accuracy with which the specific location of the mobile station was determined. 
This result means that the two anchor nodes (two EVK1000 boards) gave an accuracy figure of location finding of the mobile station that was better than that obtained in the case where three anchor nodes (EVK1000 boards) were used for the same purpose. The Ultra-Wide Band (UWB) was utilize in the real indoor localization system and the DecaWave EVK1000 evaluation board was fairly suitable for the end purpose. The (TDOA) method was successfully applied for object localization estimation in this system.

\section{REFERENCES}

Albaidhani, A., A. Morell and J.L. Vicario, 2016. Ranging in UWB using commercial radio modules: Experimental validation and NLOS mitigation. Proceeding of the 2016 International Conference on Indoor Positioning and Indoor Navigation (IPIN), October 4-7, 2016, IEEE, Alcala de Henares, Spain, ISBN:978-1-5090-2426-1, pp: 1-7.

Ali, A.A. and A.S. Omar, 2005. Time of arrival estimation for WLAN indoor positioning systems using matrix pencil super resolution algorithm. Proceedings of the Joint 2nd and 1st Workshop on Positioning, Navigation and Communication and Ultra-Wideband Expert Talk (Wpnc'05) and (Uet'05) Vol. 5, March 17, 2005, University of Hannover, Hanover, Germany, pp: 11-20.

Anonymous, 2004. APS006 application note: Channel effects on communications range and time stamp accuracy. DecaWave Ltd., Europe.
Anonymous, 2015. DW1000 user manual. DecaWave Lt, https://doc.openrtls.com/User Manual WIP SPI.pdf

Fuchs, C., N. Aschenbruck, P. Martini and M. Wieneke, 2011. Indoor tracking for mission critical scenarios: A survey. Pervasive Mob. Comput., 7: 1-15.

Gezici, S., 2008. A survey on wireless position estimation. Wirel. Pers. Commun., 44: 263-282.

Liu, H., H. Darabi, P. Banerjee and J. Liu, 2007. Survey of wireless indoor positioning techniques and systems. IEEE Trans. Syst. Man Cybern. Part C: Appl. Rev., 37: 1067-1080.

Pahlavan, K., X. Li and J.P. Makela, 2002. Indoor geolocation science and technology. IEEE Commun. Mag, 40: 112-118.

Perttula, A., H. Leppakoski, M. Kirkko-Jaakkola, P. Davidson and J. Collin et al., 2014. Distributed indoor positioning system with inertial measurements and map matching. IEEE. Trans. Instrum. Meas., 63: 2682-2695.

Yan, J., 2010. Algorithms for indoor positioning systems using ultra-wideband signals. Master Thesis, Delft University of Technology, Delft, Netherlands.

Yayan, U. H. Yucel and A. Yazici, 2015. A low cost ultrasonic based positioning system for the indoor navigation of mobile robots. J. Intell. Rob. Sys., 78: $541-552$.

Zekavat, R. and R.M. Buehrer, 2011. Handbook of Position Location: Theory, Practice and Advances. John Wiley \& Sons, New Jersey, USA., ISBN:9780470943427, Pages: 1264. 